\title{
Neurosyphilis Presenting as Intermittent Explosive Disorder and Acute Psychosis
}

\author{
Harneel S. Saini ${ }^{1}$, Matthew Sayre ${ }^{2}$, Ishveen Saini ${ }^{3}$, Nehad Elsharkawy ${ }^{4}$ \\ 1. Neurology, Allegheny General Hospital, Pittsburgh, USA 2. Internal Medicine, Lewis Katz School of Medicine at \\ Temple University, Philadelphia, USA 3. Internal Medicine, Lake Erie College of Osteopathic Medicine (LECOM), Erie, \\ USA 4. Pharmacy, Lake Erie College of Osteopathic Medicine (LECOM), Bradenton, USA
}

Corresponding author: Harneel S. Saini, neelneurologist@gmail.com

\begin{abstract}
We present a case of a patient with tertiary syphilis, manifesting as acute psychosis, auditory hallucinations and intermittent explosive disorder with pending legal ramifications for physical violence. Our patient had been seen and treated by a psychologist with Aripiprazole for his erratic and aggressive behavior coupled with his new found psychosis over a one-year period with no avail. Prior accounts of interaction with the patient described him as "easy going", "laid back", and cooperative. Our patient had a complete return to baseline mentation and functionality post treatment with 4 Million Units every four hours of penicillin for two weeks. Neurosyphilis is a disease that greatly affects the mental functioning capacity of those infected. While treatment of syphilis has become greatly straightforward, those living in impoverished conditions and without a continual access to the health care system can progress through the stages of syphilis. It is of vital importance to keep syphilis on our differential for patients with rapidly progressing and broadly encompassing psychiatric disturbances especially in patients that have a lower socioeconomic status.
\end{abstract}

Categories: Neurology, Infectious Disease, Public Health Keywords: syphilis

\section{Introduction}

Syphilis is a sexually transmitted disease caused by the gram-negative bacteria, Treponema pallidum. In structure, it is seen as a spiral microbe that is capable of causing debilitating effects on those infected such as certain neurological disorders and even death. Syphilis is observed in three stages categorized by varying levels of intensity [1]. Primary syphilis is usually observed as a single painless sore known as a chancre. This sore heals on its own within a couple of weeks. Secondary syphilis is seen as a rash that starts on the trunk of the body that eventually spreads across the entire body. The patient may experience fever, sore throat and fatigue [2]. If the patient is not treated properly for syphilis, the infection may become latent in which no symptoms are seen, but may continue to worsen and progress into the tertiary stage. Those infected in the tertiary stage may have damage caused to the brain, nerves, liver, and other organs. This stage is

Received 09/19/2019

Review began 10/20/2019 Review ended 12/05/2019 Published 12/10/2019

\section{() Copyright 2019}

Saini et al. This is an open access article distributed under the terms of the Creative Commons Attribution License CC-BY 3.0., which permits unrestricted use, distribution, and reproduction in any medium, provided the original author and source are credited. characterized by the bacterium infecting vital organs and can present as difficulty controlling voluntary muscle movement, vision problems and even dementia [1].

Perhaps the most debilitating and life altering outcome of an untreated syphilis infection is neurosyphilis in which the brain and spinal cord are affected. There are five classifications of neurosyphilis: asymptomatic, meningeal, meningovascular, general paresis and tabes dorsalis [1,2]. Asymptomatic neurosyphilis is the most common type in which no signs of neurological disease are seen. In meningeal neurosyphilis, the patient may experience stiff neck, nausea, and headaches as well as hearing loss or loss of vision. On the more critical end of the spectrum, meningovascular neurosyphilis is diagnosed if the patient experiences at least one stroke. The fourth classification of neurosyphilis, general paresis, can manifest as dementia, changes in personality and mood, weakening muscles, fatigue and paresis. Tabes dorsalis is diagnosed when the infection affects the spinal cord subsequently manifesting as ataxia, sensory and other motor disturbances [1-3].

\section{Case Presentation}

A 31-year-old Caucasian male presented to the emergency department in Northeastern Ohio, complaining of confusion and seeking treatment for a recently confirmed diagnosis of neurosyphilis. The patient's new girlfriend, a healthcare professional, had recently been diagnosed with syphilis and hepatitis $\mathrm{C}$, subsequently prompting the patient to get tested. As part of his diagnostic workup, the patient underwent a lumbar puncture and CSF-VDRL test (cerebrospinal fluid-venereal disease research laboratory test) resulting in a rapid plasma reagin (RPR) titer of 1:64 and VDRL titer of 1:256 which means he tested positive for Syphilis. He was sent to a nearby hospital for further treatment. After receiving one dose of 4 Million Units of intravenous penicillin, the patient became acutely agitated demanding to smoke. He was denied and subsequently became furious, lashed out at the faculty and left the hospital against medical advice. Early next morning, he presented to our hospital accompanied by his girlfriend in a combative and confused state. Upon questioning, the patient reported he had been diagnosed with intermittent explosive disorder with 
psychosis four months prior to current admission and was prescribed Aripiprazole. He also described a new onset of hearing voices which he described had no meaning or direction just a montage of irrelevant voices. He was particularly distressed by himself because he described himself as "calm" and "nice" but now could no longer control his anger and violent outbursts. On presentation he had a diffuse upper back and left lateral forearm maculopapular and non-blanching rash. Other symptoms included headache, fever, night sweats, $50 \mathrm{lb}$ weight loss over three months, agitation, psychosis, neuropathy, diffuse tender lymphadenopathy and flank pain. He had penile and oral lesions several months ago that had resolved by the time of admission. The patient had a past medical history significant for intravenous drug use (IVDU) and 20 pack-year smoking history. The patient denied nausea, vomiting, diarrhea and constipation. On physical exam, the patient was afebrile, had cranial nerves 2 to 12 grossly intact, pupils accommodated and reacted bilaterally, no nystagmus present, muscle strength $5 / 5$, deep tendon reflexes $2 / 4$ and sensation bilaterally intact upper and lower extremities, no ataxia, negative dysdiadokinesia and finger to nose bilaterally. Heart and lungs were normal - no murmur, point of maximal intensity (PMI) was nondisplaced and no wheezes, crackles or rhonchi were auscultated. The patient had a negative HIV screen but a positive Hep C panel. Although MRI might have provided additional information, the patient was not agreeable to the testing. The patient was discharged, threatening to leave against medical advice (AMA), on IV penicillin G 4 Million Units every four hours for two weeks administered by his girlfriend and he was taken off the Aripiprazole. The patient and his girlfriend reported resolution of aggressive behavior and auditory hallucinations with return to baseline mentation.

\section{Discussion}

Syphilis is known as the "The Great Imitator" because of its ability to mimic the symptoms of many other diseases. As a result, neurosyphilis can be a challenging diagnosis to make due to the overlap of symptoms and similarity of clinical features to other psychiatric disorders. This deception of presentation makes it no surprise that it is oftentimes misdiagnosed. Schizophrenia, dementia, and bipolar disorders, are all diagnoses that have found their way onto the diagnostic differential in a patient with neurosyphilis. In a study published by Lin et al., of 169 neurosphyilis patients the study recruited, 52 of the patients presented with psychiatric manifestations. These included cognitive impairment, personality disorders, delirium, hostility, dysarthria, paranoia, fecal and urinary incontinence, hallucinations and mania [4]. In 1992, Roberts and Emsley described neurosyphilis presentations. The most common presenting symptoms in patients of neurosyphilis were personality change in $76 \%$ of patients, memory dysfunction (62\%), hostility (52\%), hallucination (48\%), delusion (19\%), and interestingly enough only $12 \%$ of the patients had the classic neurological symptoms of Marcus Gunn decreased vibratory, motor weakness and ataxia. Diagnosis of "confirmed" neurosyphilis then relies on presentation of any stage of syphilis and a reactive CSF-VDRL [5]. Although the patient's CSF analysis and clinical picture are consistent with a diagnosis of neurosyphilis, a coexisting psychiatric disorder (previous diagnosis of intermittent explosive disorder with psychosis) or a drug-induced psychosis/withdrawal syndromes (history of IVDU) could not be ruled out. The patient was being treated with Abilify for his diagnosed psychiatric disorder and during the reported admission, the patient remained aggressive, and indicated that he was also hearing voices. Personality changes and auditory hallucinations are all documented associations of late neurosyphilis, and their presence indicates that the disease itself has been present for a long period of time [6-7].

There is no consensus on treatment regimens for patients with neurosyphilis and psychotic features in terms of which neurotropic agents to use and at what dose. Hung et al. found that treatment with penicillin alone resolved their neurosyphilis (NS) patient's auditory hallucinations and religious delusions [8]. In a case report documenting a 40-year-old male positive for neurosyphilis, antipsychotic treatment was attempted but was not found to be a beneficial measure [9]. However, in another case report $400 \mathrm{mg}$ of valproate twice a day was used to reduce agitation symptoms effectively [10]. Of note, in the latter case study, penicillin was not effective in completely clearing the patient of psychotic symptoms. This unfortunate case may be indicative that some of those who eventually reach the state of neurosyphilis may never fully recover from the psychotic symptoms without additional antipsychotic medications. There have also been many cases described of using both anti-psychotic medications in combination with antibiotics. Sanchez and Zisselman described a series of five case reports of neurosyphilis patients treated effectively with anti-psychotics in combination with antibiotics [11-14]. Interestingly enough, our patient found Haloperidol suppressed his irritation and psychosis adequately and repeatedly requested it to help with masking his symptoms. Eventually our patient was taken off his prescription of Aripiprazole and appeared to have resolved his psychotic with penicillin alone.

\section{Conclusions}

Syphilis is a sexually transmitted disease that has continued to be a challenge for health care providers. The World Health Organization estimates the incidence of new syphilis cases to be 6.1 million per year worldwide. Although syphilis can occur in stages, its spread to the central nervous system (CNS) can occur early or late in the disease. We present this case as a reminder of how important it is to consider neurosyphilis symptomatology even in a patient with a previous psychiatric diagnosis. The effects of delaying appropriate treatment or noncompliance from the patient can result in devastating effects and may even lead to death. 


\section{Additional Information \\ Disclosures}

Human subjects: Consent was obtained by all participants in this study. Conflicts of interest: In compliance with the ICMJE uniform disclosure form, all authors declare the following: Payment/services info: All authors have declared that no financial support was received from any organization for the submitted work. Financial relationships: All authors have declared that they have no financial relationships at present or within the previous three years with any organizations that might have an interest in the submitted work. Other relationships: All authors have declared that there are no other relationships or activities that could appear to have influenced the submitted work.

\section{References}

1. Kitayama K, Segura ER, Lake JE, et al.: Syphilis in the Americas: a protocol for a systematic review of syphilis prevalence and incidence in four high-risk groups, 1980-2016. Syst Rev. 2017, 6:195. Accessed: October 1, 2019: https://www.ncbi.nlm.nih.gov/pubmed/29017552. 10.1186/s13643-017-0595-3

2. Newman L, Rowley J, Vander Hoorn S, et al.: Global estimates of the prevalence and incidence of four curable sexually transmitted infections in 2012 based on systematic review and global reporting. PLoS One. 2015, 10:e0143304. 10.1371/journal.pone.0143304

3. Gonzalez H, Koralnik IJ, Marra CM: Neurosyphilis. Semin Neurol. 2019, 39:448-455. Accessed: October 1, 2019: https://www.thieme-connect.de/products/ejournals/abstract/10.1055/s-0039-1688942. 10.1055/s0039-1688942

4. Lin LR, Zhang HL, Huang SJ, et al.: Psychiatric manifestations as primary symptom of neurosyphilis among HIV-negative patients. J Neuropsychiatry Clin Neurosci. 2014, 26:233-240.

5. Roberts MC, Emsley RA: Psychiatric manifestations of neurosyphilis. S Afr Med J. 1992, 82:335-337.

6. Ghanem KG: Neurosyphilis: a historical perspective and review. CNS Neurosci Ther. 2010, 16:157-168. 10.1111/j.1755-5949.2010.00183.x

7. Danielsen AG, Weismann K, Jorgensen BB, Heidenheim M, Fugleholm AM: Incidence, clinical presentation and treatment of neurosyphilis in Denmark 1980-1997. Acta Derm Venereol. 2004, 84:459-462. 10.1080/00015550410017308

8. French P: Syphilis. BMJ. 2007, 334:143. 10.1136/bmj.39085.518148.BE

9. Hung HC, Chu JL, Lu T: Psychotic disorder due to neurosyphilis. J Neuropsychiatry Clin Neurosci. 2013, 25:67-68. 10.1176/appi.neuropsych.12090217

10. Wahab S, Md Rani SA, Sharis Othman S: Neurosyphilis and psychosis. Asia Pac Psychiatry. 2013, 5:90-94. 10.1111/appy. 12050

11. Tibrewal P, Kumar I, Zutshi A, Math SB: Valproate for treatment of agitation in neurosyphilis: a case report . Prim Care Companion J Clin Psychiatry. 2008, 10:163. 10.4088/pcc.v10n0213d

12. Sanchez FM, Zisselman MH: Treatment of psychiatric symptoms associated with neurosyphilis . Psychosomatics. 2007, 48:440-445. 10.1176/appi.psy.48.5.440

13. Kolokolov O, Bakulev A, Sholomov I, et al.: "Early" and "late” neurosyphilis: the historical background and the current look at the issue. J Neurol. 2011, 3:13.

14. Klausner JD: The great imitator revealed: syphilis. Top Antivir Med. 2019, 27:71-74. Accessed: October 1, 2019: https://www.ncbi.nlm.nih.gov/m/pubmed/31136995/?i=46\&from=syphilis \&sort=[relevance]. 